\title{
Studying and Analyzing of one of Gaudi's Most Popular Works (Casa Batallo)
}

\author{
Mrs. Najlaa Yousif \\ Department of Architecture and Interior Design, University of Bahrain
}

\begin{abstract}
It is possible to discuss Gaudís various productions and to study them from a certain perspective, not only within the boundaries of personal interests in those works from the structural aspect, but also due to that being a pointer for solving current problems $\mathrm{s}^{\mathrm{ii}}$. When inspecting those works we may identify the concepts that all of Gaudís productions are based on. In fact, many architects today, especially those interested in the topics of the new pioneer movement, in addition to the general public, have shown increasing interests, as Gaudí's connecting points are linked with the majority of studies today in many countries. They have noticed, for example, the role of manual production and participation themes. In addition, the aesthetic research results for Gaudí contain many motivations that we feel that are still considered to be fertile, which lead us to rediscover him again and again. We shall discover that there is a renewable request to this manual artistic aspect, or in other words, the manual culture that can be observed in many fields of design. Now we will studying one of the most important works by Gaudi in architecture and interior design (Casa Batallo).
\end{abstract}

Keywords: Gaudí's, Architecture, Interior Design, Casa Batallo, Manual Culture.

\section{INTRODUCTION}

Casa Batlló represents a reformation of the façade of a Gaudí was assigned to this task by José Batlló, where he very interesting building that was built in 1875 in Paseo was ordered to transform the façade of the building to a De Gràcia, which was considered at that time to be the modern and attractive one, in addition to preparing the most prestigious street in Barcelona.

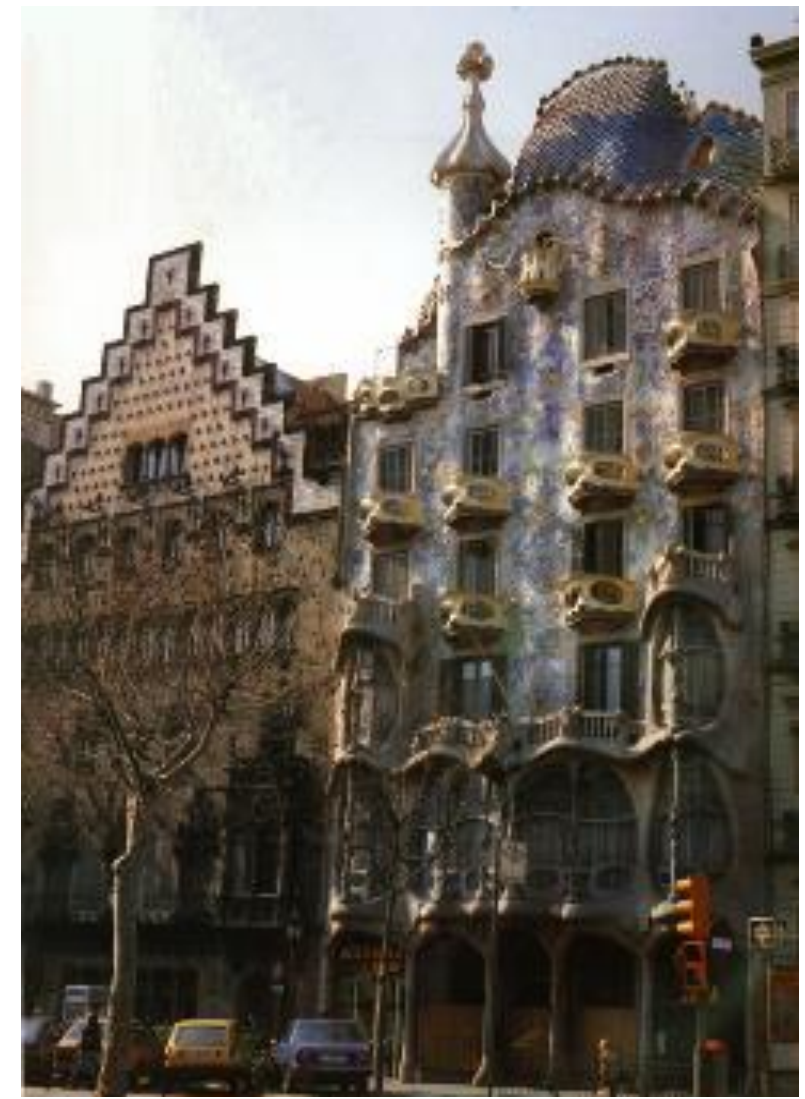

Figure No 1 modern and attractive one, in addition to preparing the
main floor for the usage of the owner's family as a residence.

Furthermore, he was ordered to modernize the rest of the building in order to lease it as residential units, while the ground floor was worked on to be used as offices. In addition to that, the owner asked him to make Casa Batlló greater than Casa Amatller, which was next to it and build by( Puig i Cada P. fach) in 1900, Figure No 1

\section{EXTERNAL DESCRIPTION OF THE BUILDING}

The original façade of Casa Batlló consisted of four floors, each with four rectangular balconies. In order for Gaudí to get rid of this regularity, he created five stone arches on the façade of the ground floor that led to giving the first floor a continuous platform upwards on every edge of the building's two edges.

$\mathrm{He}$ added a fifth floor to be used as a residence for servants. Following that, he added a ceiling made in the form of a dragon's back, filling with bricks that look like scales or a curved backbone. The small tower in the center was planned for in the original design but it was moved to the side during the construction phase, Figure No 2.

Gaudí was able to create a balcony behind a small gap in the ceiling above the entrance. Through this gap, residents could see below without anyone from outside being able to see them. 


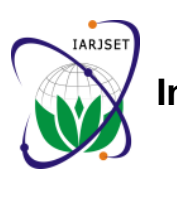

IARJSET

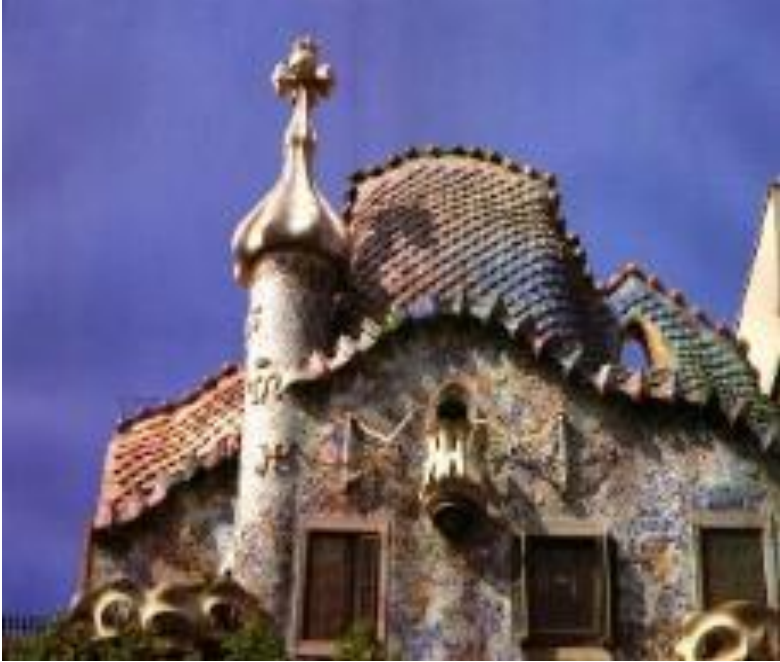

Figure No 2 Façade of Casa Batlló

The most interesting element in the façade is the existing of a central window with two side windows each split by a central pillar that looks like bones, Figure No 3.

The top wall of the façade is filled with blue and green ceramic decorated with circles of colored ceramic. The designer confirmed using decoration raw materials that are suitable with the Spanish environment due to the fact that this building is located on the coast, which confirms the mental strength of the designer. Furthermore, the general appearance of the curved façade and the sand dunes go well with the surrounding environment of the building.

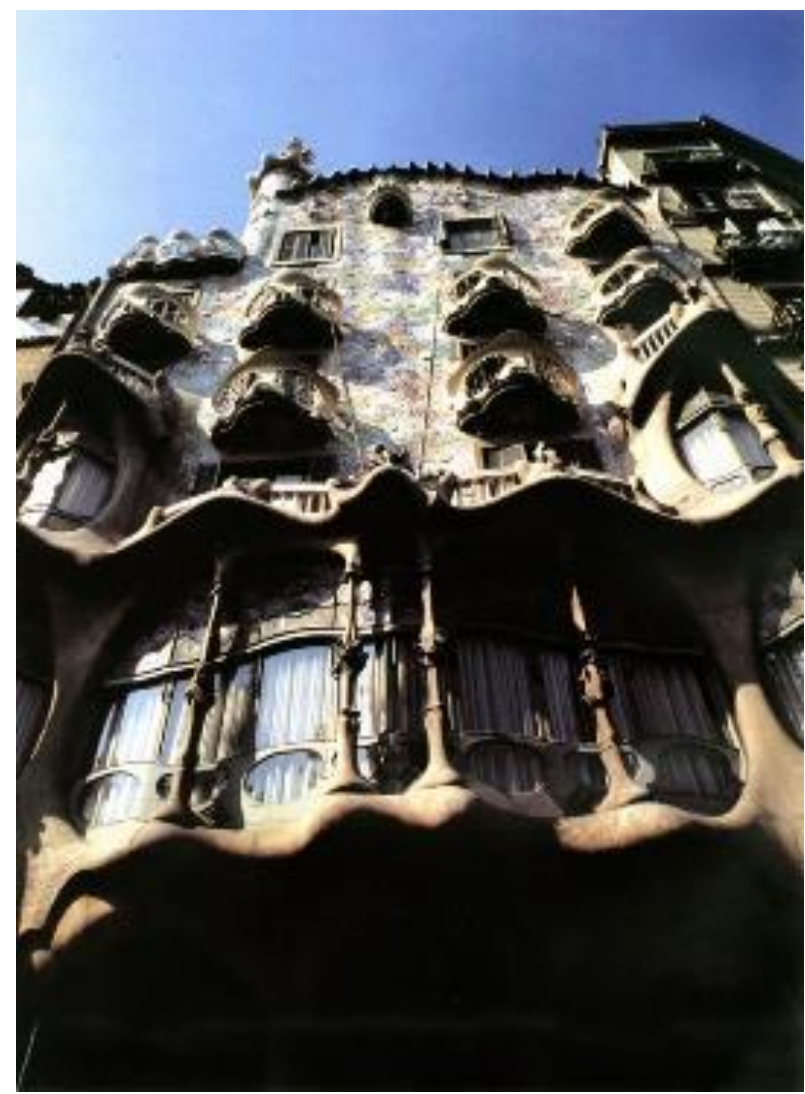

Figure No 3
I will address the building analysis through the following design elements: -

\section{1-2Appearance:}

- All in all, the façade gives an impression that emulates the forms of fictional sea creatures, which were implemented in the structural elements to confirm this feeling.

- The building in general has a main rectangular façade, above its bottom parts there are several circular arches . There are balconies above them that have curved forms to the outside which give a connected curved appearance. They are two towers to break the rhythm and to add vitality to the design.

- The rooftop of the façade in general is curved and filled with colored ceramic parts in asymmetric forms. The building's balconies were processed with crusty covering in the form of symmetric and repeated circular units.

- On top of the building, there is an irregular summit that matches the backs of dinosaurs processed with blue and green colored ceramic parts in forms overlapped by external linings with repeated protrusions.

\section{2-2 Function (Functional Fitting):}

A residential building consisting of five floors suitable for family living. It contains all services and living requirements.

- It was taken into account that the building would be compatible ecologically without being dissonant with the coastal and sea environment where it is located. At first glance, it comes to mind that you are before a fictional sea creature. The designer confirmed that by using elements and architectural solutions that emulate sea creatures regarding their appearance, coloring, surface feeling, and units. He used those to serve the functional aspect of the building.

\section{2-3 Character:}

This building confirms the design thoughts of Gaudí and his style (Art Nouveau) based on the following:

- Exiting from traditional designing.

- The general thought being affected by the Rococo style that emerged in Europe, where attention is paid to repeated decoration details.

- The building's environmental fitting and ecological compatibility.

- Employment of architectural design elements in the internal space.

\section{2-4 Materials:}

The façade is covered with blue and green ceramic, and decorated with colored circles of ceramic. Iron was also used in the formation of external windows of the building, Figure No 4 


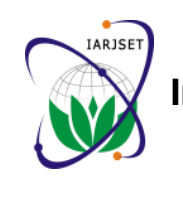

IARJSET

\section{International Advanced Research Journal in Science, Engineering and Technology}

ISO 3297:2007 Certified

Vol. 3, Issue 11, November 2016

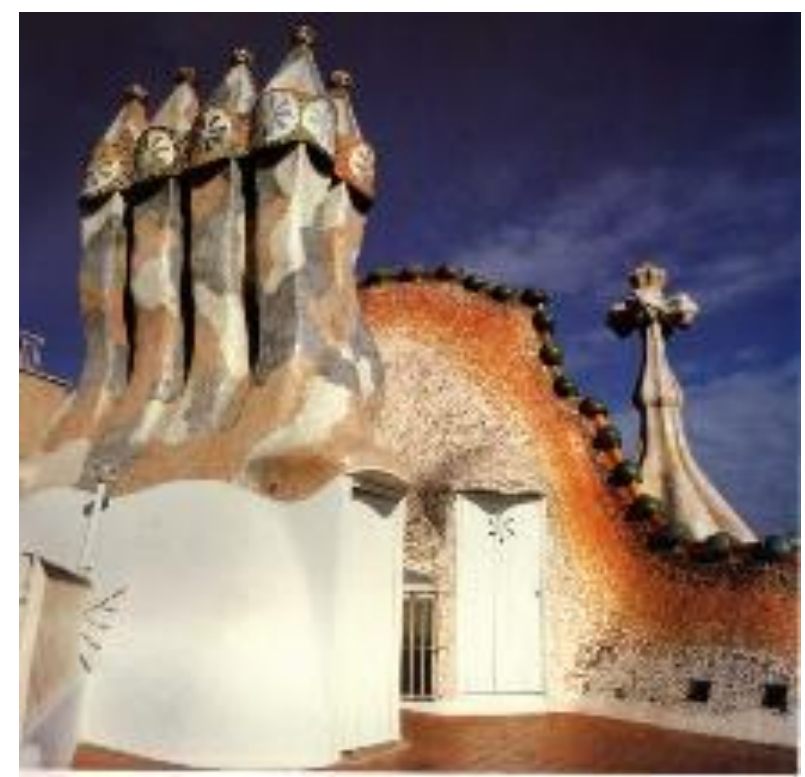

\section{INTERIOR DESIGN (INTERIOR ARCHITECTURE)}

\section{3-1 Introduction:}

The decoration of the interior space of the building reflects the beauty values of the architectural coverage and confirms the decoration appearances and the beauty relationships with them. The interior space mainly presents harmony and consensus between the positive and negative design blocs.He confirmed the values of harmony, relay, rhythm, symmetry, volumetric relations, and imparted the external atmosphere internally through some architectural gaps. Furthermore, the designer was keen to perform unity in the design, and to enforce the main theme by repeating the design unit everywhere (externally and internally) so that the external appearance does not get separated from the internal space. He confirmed that in all internal design elements, Figure No 5

Figure No 4

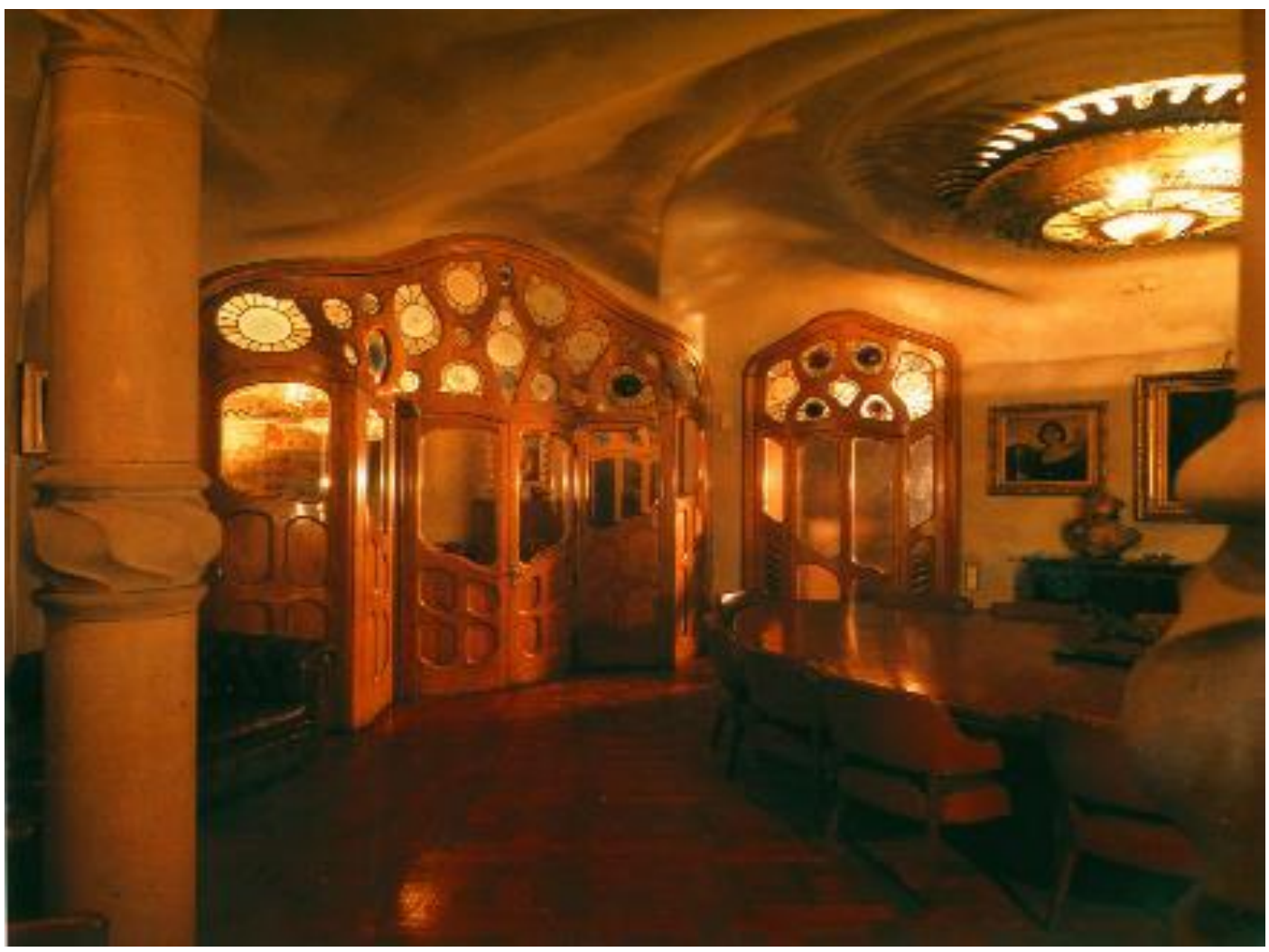

Figure No 5 the interior design of the main hall in Casa Batlló

3-2 The interior design of the main salon in Casa
Batallo
I will address the building analysis through the following

design elements:

-Appearance and Shape

-Masses and space

-Measurements and Scale

-Surfaces and Texture

-Coloring

\section{3-2-1 Appearance and Shape:}

The designer used a solution for the internal space surfaces in a completely connected way that gives one perspective for the entire vertical surfaces of the internal space. We shall not disregard surfaces occupied in gaps (such as doors and windows) that take the form of processions with 


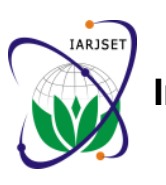

IARJSET

Vol. 3, Issue 11, November 2016

wooden leaves in the form of wall curves, where the stylish line's continuity does not get interrupted. They were formed as irregular and repeated circles. He avoided sharp forms so that they don't go well with the designing style.

\section{3-2-2 Masses and Space:}

The relation between masses and space, or between projections and niches, in a way that repeats the unit or the applied style in the building's façade design, where the designer avoided sharp masses (negative or positive) and immediate transfer between positivity and negativity.

Therefore, the space generally represented smooth curved lines gradually transferring between positivity and negativity in continuous and repeated curved lines with circular arch joints that internally add the descriptions and vitality of the exterior design, confirming harmony and specific rhyme for the internal space.

The designer did not strictly separate between the levels of walls and ceilings in his work, but instead, he merged the relations between them confirming the elemental relation between the formation elements of the internal space.

Walls came in curved forms to repeat the same lining style of the ceiling. The ground acted as a surface level and a background that shows the formation relation and this space dialog illustrated between walls and ceilings, Figure No 6.

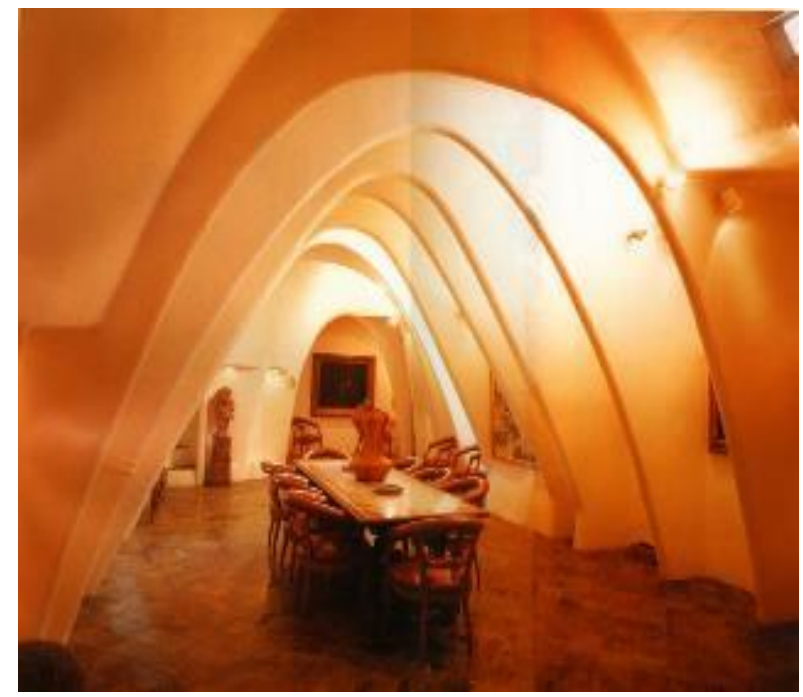

Figure No 6

The interior design of the dining room in Casa Batlló

\section{3-2-3 Measurements and Scale:}

The designer did not pay attention to achieve human measurements in the design entirely. This was followed in all European styles in the middle ages, where the interior space was distinguished in being very high and where humans would not fit divisions suitable with the measurements in order to achieve enormousness and imparted greatness in the design, Figure No 7.

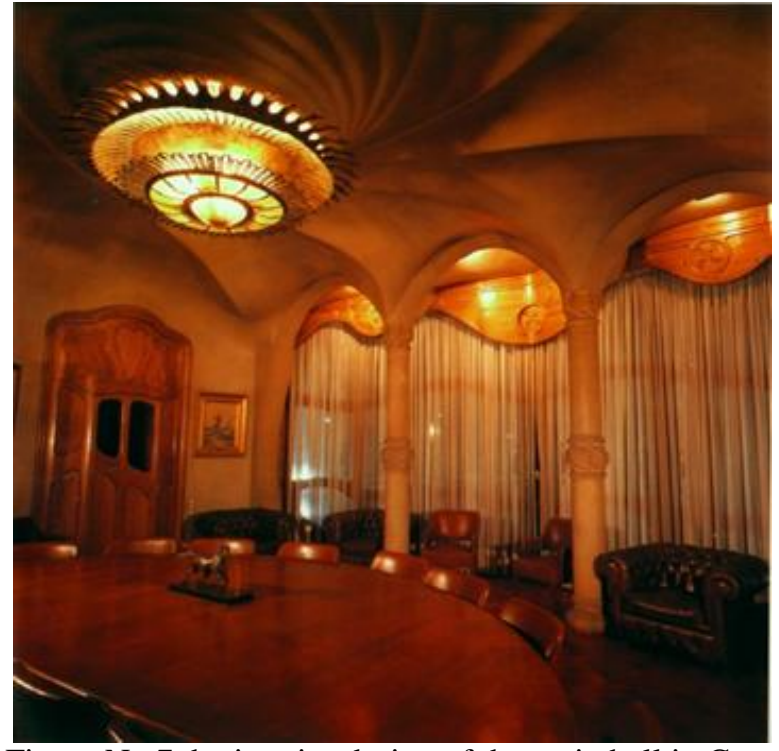

Figure No 7 the interior design of the main hall in Casa Batlló

\section{3-2-4 Surfaces and Texture:}

Processing of the surfaces generally took the form of hard surfaces that give some self-shading and lighting spots, which confirm the age of the design and that the designer avoiding finding varieties for the surface feelings but instead he focused on using one only to show the rest of the fine values of the various design elements. That entirely goes well with the path that the designer is following in his work. It also emulates some of the natural surface feelings found in environments (such as animal or fish skins, and so forth).

\section{3-2-5 Coloring:}

The designer used the beige color (yellow ochre) in coloring the entire vertical and horizontal surfaces (ceilings and walls) in the interior space which represented a calm background to illustrate the other design elements (ladders, doors, windows, furniture).

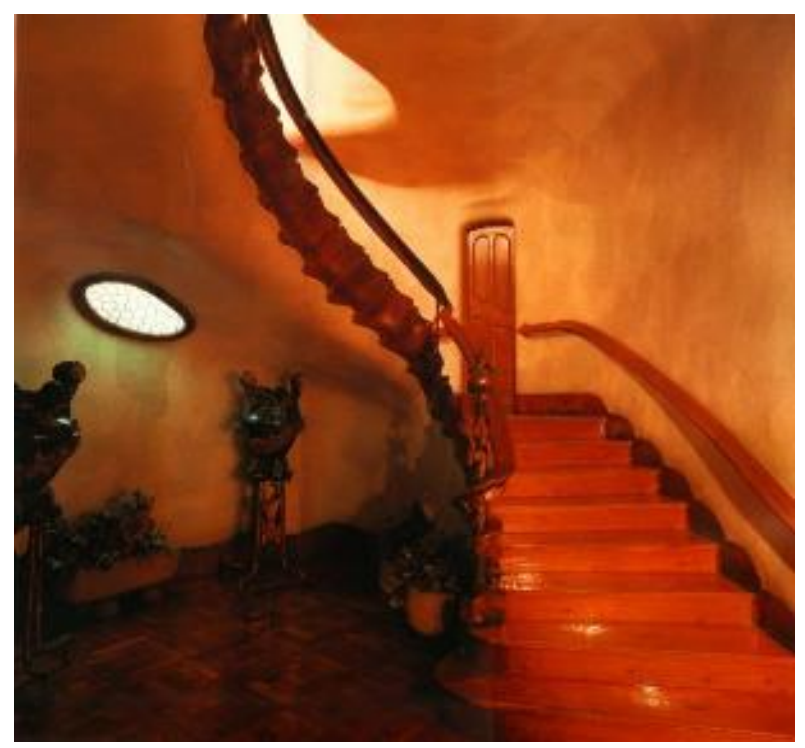

Figure No 8 
Vol. 3, Issue 11, November 2016

Grounds were processed with wooden parquet in dark colors using walnuts and oak woods in decoration formulations that are various and repeated so that they work as a splitting line for the walls in the design.

As for ladders, windows, and doors, they were formulated with light and dark brown coloring in opposite forms confirming the element of opposition in design that added vitality and confirmed the various design masses, Figure No 8 .

\section{3-2-6 Shades and Lights:}

Due to using volumetric design dialogues in the interior space, very beautiful and varied fine values were shown that use opposition techniques in the areas of shades and lighting, which added another dimension to the element of coloring confirming the masses and space appearances. The variation principle was shown, which is considered to be a basic principle of fine and architectural formulations.

\section{3-2-7 Style:}

- The designed followed the famous style of Art Nouveau, where he was affected by the previous decorative fine formulations.

- Trying to find out a relation between the architectural coverage of the building and what is included within the interior space.

- Focusing on finding out an ecological relation and an environmental dimension with all interior design elements whether that is in the form of honest emulation or emulation of the design contents.

\section{3-2-8 - Proportionality:}

The principle of proportionality is linked to the dimensional relations of the geometric shapes that form the design, such as the shapes of triangles, squares, rectangles, and circles, the relation between their two dimensions, and the relation between every shape and another. In addition, this principle is related to the portions of matters and structural elements of the design to each other or to other elements, or in other words, the portion of matter to matter, and the portion of matter to shape. In the design of this building, we will address the fine relations of the interior design between the dimensions of gaps and their heights, and as for columns. This design follows the classic proportions taken from the old classic architecture.

\section{CONCLUSION}

To conclude, Gaudi's work is characterized by its value in the design, as well as the enrichment of the architectural and the interior design formation. With the knowledge that there is an urgent need for real participation of learning this processes of his works. His works is the most important that must be considered as one of the wonderful works of art. Gaudi gave us lots of attention to the architectural works and miniature pieces of furniture that was intended to be used. We can identify that Gaudi is free at his work, as he works with handmade techniques that are very difficult to be repeated. We can also conclude, that Gaudi's work always aim to look like animals, as we see from the designs of the casa batllo for example, it looks like a dragon. Despite that, Gaudí was not described as monotonous, even when it comes to choosing the type of materials that have the highest level of purity and simplicity. Instead, he further showed excellence in great freedom during his work, even regarding usage of complicated and complex manual techniques. Previously we can conclude that, it is important to recognize the work of pioneers of architecture and benefit from their work at finding solutions to the current problems of design in architechture,interior design and furniture design solution.

\section{REFERENCES}

[1] https://www.casabatllo.es/en/history/casa-batllo/

[2] http://www.gaudidesigner.com/uk/casa-batllo.html

[3] https://www.bluffton.edu/homepages/facstaff/sullivanm/spain/barce lona/gaudibatllo/batllo.html

[4] http://www.atlasobscura.com/places/casa-batllo

[5] https://www.casabatllo.es/en/news/casa-batllo-deciphers-hiddensymbolism-gaudi-discovers-original-furniture/

[6] https://www.casabatllo.es/en/antoni-gaudi/casa-batllo-chair/

[7] https://www.casabatllo.es/en/category/antoni-gaudi/works/

[8] https://en.wikipedia.org/wiki/Confidant_from_the_Batll\%C3\%B3 House

[9] https://www.casabatllo.es/en/casa-batllo/facade-building-wellrestoration/

[10] https://en.wikipedia.org/wiki/Antoni_Gaud\%C3\%AD

[11] https://www.casabatllo.es/en/antoni-gaudi/

[12] http://www.archdaily.com/tag/antoni-gaudi

[13] Collection- Architecture ducontre Gorges Pomidou, Aianin Guiheux, 1998

[14] Maria Costantino "Gaaudi” Bromton Books Crop 1993.

[15] 'James Johnson And Joseph Luis "Antoni Gaudi The Architectural Press" London 1973. 